\title{
GLOBAL DIMENSION OF DIFFERENTIAL OPERATOR RINGS
}

\author{
K. R. GOODEARL
}

\begin{abstract}
This paper is concerned with finding the global homological dimension of the ring of differential operators $R[\theta]$ over a differential ring $R$ with a single derivation. Examples are constructed to show that $R[\theta]$ may have finite dimension even when $R$ has infinite dimension. For a commutative noetherian differential algebra $R$ over the rationals, with finite global dimension $n$, it is shown that the global dimension of $R[\theta]$ is the supremum of $n$ and one plus the projective dimensions of the modules $R / P$, where $P$ ranges over all prime differential ideals of $R$. One application derives the global dimension of the Weyl algebra over a commutative noetherian ring $S$ of finite global dimension, where $S$ either is an algebra over the rationals or else has positive characteristic.
\end{abstract}

1. Introduction. Throughout this paper, the term differential ring will refer to an associative ring $R$ with unit together with a specified derivation $\delta$ on $R$. The ring of differential operators over $R$ (also called the Ore-extension of $R$ with respect to $\delta$ ) is additively the group of polynomials over $R$ in an indeterminate $\theta$, with multiplication subject to the requirement $\theta a=$ $a \theta+\delta a$ for all $a \in R$. We denote this ring by $R[\theta]$, or by $(R, \delta)[\theta]$ if $\delta$ needs to be emphasized.

Most arguments involving right-hand properties of $R[\theta]$ can be used, with appropriate changes of sign, for the corresponding left-hand properties. The precise relationship is given by the following proposition, whose proof is routine.

Proposition 1. Let $R$ be a differential ring with derivation $\delta$. If $R^{0}$ denotes the opposite ring of $R$ together with the derivation $-\delta$, then $R^{0}[\theta]$ is isomorphic to the opposite ring of $R[\theta]$.

In particular, Proposition 1 can be used to show that all of the results in

Presented to the Socie $\overline{t y}$, January 18, 1974 under the title Homological dimension of differential operator rings; received by the editors April 24, 1973. AMS (MOS) subject classifications (1970). Primary 16A60; Secondary 12H05.

Key words and phrases. Global dimension, rings of linear differential operators, differential algebra, Weyl algebras. 
this paper on the right global dimension of $R[\theta]$ carry over to the left global dimension, hence we shall not state the left-hand versions explicitly.

2. General differential rings. In considering the global dimension of $R[\theta]$ several authors [1], [3], [4], [10] have stated as "well-known" the inequalities

$$
\text { r. gl. } \operatorname{dim} . R \leq \text { r. gl. } \operatorname{dim} . R[\theta] \leq 1+\text { r. gl. } \operatorname{dim} . R \text {. }
$$

While the right-hand inequality is true in general, the left-hand one may fai. if r. gl. dim. $R$ is allowed to be infinite, and we present examples of this at the end of the section. For clarity, therefore, we now derive the valid cases of these inequalities.

Given a module $A$ over a ring $S$, we use $\operatorname{pd}_{S}(A)$ and $\operatorname{wd}_{S}(A)$ to denote the projective and weak dimensions of $A$.

Proposition 2. Let $R$ be a differential ring. If $A$ is any right $R[\theta]$-module, then

$$
\operatorname{pd}_{R}(A) \leq \operatorname{pd}_{R}[\theta]^{(A)} \leq 1+\operatorname{pd}_{R}(A)
$$

and

$$
\mathrm{wd}_{R}(A) \leq \mathrm{wd}_{R}[\theta](A) \leq 1+\mathrm{wd}_{R}(A) .
$$

Proof. Inasmuch as $(R[\theta])_{R}$ is free, any projective resolution for $A_{R}[\theta]$ is also a projective resolution for $A_{R}$, whence $\operatorname{pd}_{R}(A) \leq \operatorname{pd}_{R}[\theta](A)$. As in the case of polynomials [6, Part III, Theorem 6], there exists an exact sequence $0 \rightarrow A \otimes_{R} R[\theta] \rightarrow A \otimes_{R} R[\theta] \rightarrow A \rightarrow 0$ of right $R[\theta]$-modules, from which we infer that $\operatorname{pd}_{R}[\theta](A) \leq 1+\operatorname{pd}_{R}(A)$.

The inequalities for weak dimension are proved in the same way.

Proposition 3. If $R$ is any nonzero differential ring, then

$$
1 \leq \text { r. gl. } \operatorname{dim} . R[\theta] \leq 1+\text { r. gl. dim. } R \text {. }
$$

In case r. gl. $\operatorname{dim} . R<\infty$, then also

$$
\text { r. gl. } \operatorname{dim} . R \leq \text { r. gl. } \operatorname{dim} . R[\theta] \text {. }
$$

Proof. The inequality r. gl. $\operatorname{dim} . R[\theta] \leq 1+\mathrm{r}$. gl. dim. $R$ is clear from Proposition 2. Observing that $R[\theta] / \theta R[\theta]$ cannot be isomorphic to a right ideal of $R[\theta]$, we see that r. gl. $\operatorname{dim} . R[\theta] \geq 1$.

Now assume that r. gl. $\operatorname{dim} . R=n<\infty$, and choose a right $R$-module $A$ with $\operatorname{pd}_{R}(A)=n$. There is an obvious exact sequence $0 \rightarrow A \rightarrow A \otimes_{R} R[\theta] \rightarrow$ $B \rightarrow 0$, and certainly $\operatorname{pd}_{R}(B) \leq n$, hence we infer from the long exact sequence 
for Ext that $\operatorname{pd}_{R}\left(A \otimes_{R} R[\theta]\right)=n$. In view of Proposition 2, it now follows that r. gl. $\operatorname{dim} . R[\theta] \geq n$.

We now give an example of a differential ring $R$ with r. gl. dim. $R=\infty$ and r. gl. $\operatorname{dim} . R[\theta]=1$.

Choose a field $F$ of characteristic 2 and consider the derivation $\partial / \partial x$ on $F[x]$. The ideal $\left(x^{2}\right)$ is a differential ideal because of characteristic 2, hence we obtain a differential ring $R=F[x] /\left(x^{2}\right)$. As an $F$-algebra, $R$ has a basis $1, w$ such that $w^{2}=0$ and $\delta w=1$. Observing an exact sequence $0 \stackrel{\rightarrow}{\rightarrow}=\rightarrow R \rightarrow w R \rightarrow 0$, we infer that gl. dim. $R=\infty$.

Given any right $R[\theta]$-module $A$, we may choose a basis $\left\{b_{a}\right\}$ over $F$ for the vector space $B=\{b \in A \mid b w=0\}$. Set $a_{a}=b_{a} \theta$ for all $a$, and note that $a_{a} w=b_{a}$. It is routine to check that $A_{R}$ is free with basis $\left\{a_{a}\right\}$, hence we obtain $\operatorname{pd}_{R}[\theta]^{(A)} \leq 1$ from Proposition 2, and thus r. gl. dim. $R[\theta]=1$.

This example may be extended somewhat as follows. Set $R_{1}=R$ as above, and for $n>1$ set $R_{n}=R\left[x_{1}, \cdots, x_{n-1}\right]$ and extend $\delta$ to a derivation of $R_{n}$ by defining $\delta x_{i}=0$ for all $i$. This choice of $\delta$ ensures that the $x_{i}$ all commute with $\theta$ in $R_{n}[\theta]$, whence $R_{n}[\theta] \cong R[\theta]\left[x_{1}, \cdots, x_{n-1}\right]$ and so r. gl. $\operatorname{dim} . R_{n}[\theta]=n$. We summarize these examples as follows:

For each positive integer $n$, there exists a commutative noetherian differential ring $R_{n}$ such that gl. dim. $R_{n}=\infty$ and r. gl. $\operatorname{dim} . R_{n}[\theta]=n$.

3. Commutative noetherian differential rings. For some of the results in this section, we require that $R$ be an algebra over the rationals, in which case we follow Kaplansky [5] in using the term Ritt algebra to denote a commutative differential ring which is an algebra over the rationals.

Lemma 4. Let $R$ be a noetherian Ritt algebra. If $A$ is any nonzero finitely generated right $R[\theta]$-module, then there exists a chain of $R[\theta]-s u b$ modules $A_{0}=0<A_{1}<\cdots<A_{k}=A$ and a collection of prime ideals $P_{1}, \ldots, P_{k}$ of $R$ such that for each $i$, either $A_{i} / A_{i-1} \cong R[\theta] / P_{i} R[\theta]$ or else $P_{i}$ is a differential ideal and $A_{i} / A_{i-1}$ is a torsion-free $\left(R / P_{i}\right)$-module.

Proof. As observed in [1, p. 68], $R[\theta]$ is right noetherian, and so $A$ has ACC on $R[\theta]$-submodules. Thus it suffices to show that there exists a nonzero $R[\theta]$-submodule $B$ of $A$ and a prime ideal $P$ in $R$ satisfying one of the two relationships described.

Choose a nonzero $x \in A$ whose $R$-annihilator $P=\{r \in R \mid x r=0\}$ is maximal among the $R$-annihilators of nonzero elements of $A$, and set $B=x R[\theta]$. According to [7, Theorem 6], $P$ is a prime ideal of $R$. If $P$ is a differential ideal, then $R[\theta] P=P R[\theta]$ and we obtain $B P=0$. In this case the maximality 
of $P$ now ensures that $B_{R / P}$ is torsion-free. Clearly $B \cong R[\theta] / J$ for some right ideal $J$ of $R[\theta]$, such that $P^{\prime} R[\theta] \subseteq J$ and $J \cap R=P$. If $P$ is not a differential ideal, then an easy argument due to Hart in [4, Lemma 2.4] shows that $J=P R[\theta]$.

Theorem 5. Let $R$ be a noetherian Ritt algebra with gl. dim. $R=n<\infty$. If $\operatorname{pd}_{R}(R / P)<n$ for all prime differential ideals $P$ of $R$, then r. gl. $\operatorname{dim} . R[\theta]$ $=n$.

Proof. If $n=0$, then $R$ must contain a prime ideal $P$ which is generated by an idempotent $e$. Differentiating the identities $e^{2}=e$ and $(1-e)^{2}=$ $1-e$, we find that $\delta e=0$ and so $P$ is a differential ideal. But $\operatorname{pd}_{R}(R / P)<0$ is impossible, hence we must have $n \neq 0$.

We have r. gl. $\operatorname{dim} . R[\theta] \geq n$ by $\mathrm{P}$ roposition 3 , hence it suffices to prove that $\operatorname{pd}_{R}[\theta](A) \leq n$ for any nonzero finitely generated $A_{R}[\theta] \cdot$. Since $R[\theta]$ is right noetherian, it is enough to show that $\operatorname{wd}_{R}[\theta](A) \leq n$. In view of Lemma 4, we may also assume that there is a prime ideal $P$ in $R$ such that either $A \cong R[\theta] / P R[\theta]$ or else $P$ is a differential ideal and $A$ is a torsion-free $(R / P)$-module.

If $A \cong R[\theta] / P R[\theta]$, then obviously $\mathrm{wd}_{R}[\theta](A) \leq \mathrm{wd}_{R}(R / P) \leq n$.

Now assume that $P$ is a differential ideal and that $A$ is a torsion-free $(R / P)$-module. Then $A_{R}$ can be embedded in some direct product $T$ of copies of the quotient field of $R / P$, and since $R / P$ is noetherian we see that $T_{R / P}$ is flat. We are given $\operatorname{pd}_{R}(R / P) \leq n-1$, and we infer from [2, Proposition 4.1.2, p. 117] that $\mathrm{wd}_{R}(T) \leq n-1$ also. There is an exact sequence $0 \rightarrow A \rightarrow T \rightarrow C \rightarrow 0$, and certainly $\mathrm{wd}_{R}(C) \leq n$, hence it follows from the long exact sequence for Tor that $\operatorname{wd}_{R}(A) \leq n-1$. By Proposition 2 , $\operatorname{wd}_{R}[\theta]^{(A)} \leq n$.

Lemma 6. Let $R$ be a commutative noetherian differential ring with gl. $\operatorname{dim} . R=n<\infty$. If $R$ is local with maximal ideal $M$, and if $M$ is a differential ideal, then r. gl. $\operatorname{dim} . R[\theta]=n+1$.

Proof. We already have r. gl. dim. $R[\theta] \leq n+1$ by Proposition 3. According to $[6, P$ art III, Theorems 12,13$], R$ has classical Krull dimension $n$, and by $[8,(\mathrm{~h})] R$ must al so have Krull dimension $n$ in the sense of [8].

Inasmuch as $M$ is a differential ideal, $R / M$ is a differential ring and $M R[\theta]$ is a two-sided ideal of $R[\theta]$.with $R[\theta] / M R[\theta] \cong(R / M)[\theta]$. Observing that the right Krull dimension of $(R / M)[\theta]$ is at least 1 , we see from $[1$, Lemma 2.c.1] that the right Krull dimension of $R[\theta]$ is at least $n+1$. 
Now $R[\theta]$, is a filtered noetherian ring whose associated graded ring is the commutative regular noetherian ring $R[x]$. According to a theorem of Roos in [11] (quoted in [1, p. 78]), the right Krull dimension of $R[\theta]$ is at most r. gl. $\operatorname{dim}$. $R[\theta]$, hence we obtain r. gl. $\operatorname{dim} . R[\theta] \geq n+1$.

Lemma 7. Let $R$ be a commutative differential ring, $S$ any multiplicative subset of $R$.

(a) $\delta$ induces a derivation on $R_{S}$ by the rule $\delta(r / s)=[(\delta r) s-r(\delta s)] / s^{2}$.

(b) The natural map $\phi: R[\theta] \rightarrow R_{S}[\theta]$ makes $R_{S}[\theta]$ into a flat left $R[\theta]$. module such that the multiplication map $R_{S}[\theta] \otimes_{R}[\theta] R_{S}[\theta] \rightarrow R_{S}[\theta]$ is an isomorphism.

(c) r. gl. $\operatorname{dim} . R_{S}[\theta] \leq$ r. gl. $\operatorname{dim} . R[\theta]$.

Proof. (a) is an easy verification.

(b) We check that $\operatorname{ker} \phi=\{x \in R[\theta] \mid x s=0$ for some $s \in S\}$, that each element of $\phi(S)$ is invertible in $R_{S}[\theta]$, and that every element of $R_{S}[\theta]$ may be written in the form $(\phi x)(\phi s)^{-1}$ for suitable $x \in R[\theta], s \in S$. Thus $R_{S}[\theta]$ is just the classical localization of $R[\theta]$, with respect to the multiplicative set $S$, and the required properties follow as in the commutative case.

(c) According to (b), $R_{S}[\theta]$ is a right localization of $R[\theta]$ in the sense of [12], hence this in equality follows from [12, Corollary 1.3].

Theorem 8. Let $R$ be a commutative noetherian differential ring with gl. $\operatorname{dim} . R=n<\infty$. If $R$ contains a prime differential ideal $P$ such that $\mathrm{pd}_{R}(R / P)=n$, then r. gl. $\operatorname{dim} . R[\theta]=n+1$.

Proof. We already have r. gl. dim. $R[\theta] \leq n+1$ by Proposition 3. According to [6, Part III, Theorem 11], gl. dim. $R_{P} \leq n$. There is a natural exact sequence $0 \rightarrow R / P \rightarrow R_{P} / P R_{P} \rightarrow A \rightarrow 0$, and we have $\operatorname{wd}_{R}(R / P)=n$, $\operatorname{wd}_{R}(A) \leq n$, hence it follows from the long exact sequence for Tor that $\operatorname{wd}_{R}\left(R_{P} / P R_{P}\right)=n$. Because $\left(R_{P}\right)_{R}$ is flat, the weak dimension of $R_{P} / P R_{P}$ over $R_{P}$ must be at least $n$, and we conclude that gl. $\operatorname{dim} . R_{P}=n$.

Now $R_{P}$ is a differential ring as in Lemma 7, and $P R_{P}$ is a differential ideal of $R_{P}$ because $P$ is a differential ideal of $R$, hence we obtain r. gl. $\operatorname{dim} . R_{P}[\theta]=n+1$ from Lemma 6. Therefore r. gl. $\operatorname{dim} . R[\theta] \geq n+1$, by Lemma 7 .

Corollary 9. Let $R$ be a commutative noetherian differential ring with gl. $\operatorname{dim} . R=n<\infty$, and assume that $R$ has prime characteristic $p$. If $R$ contains a prime ideal $Q$ such that $\delta^{p}(Q) \subseteq Q$ and $\operatorname{pd}_{R}(R / Q)=n$, then r. gl. $\operatorname{dim} . R[\theta]=n+1$. 
Proof. Because of characteristic $p$, it follows from Leibnitz' rule that $\delta^{p}$ is a derivation on $R$. Likewise, we obtain $\theta^{p} a=a \theta^{p}+\delta^{p} a$ for all $a \epsilon$ $R$, whence the set $R\left[\theta^{p}\right]$ is a subring of $R[\theta]$ isomorphic to $\left(R, \delta^{p}\right)[\theta]$. Since $Q$ is a prime differential ideal in $\left(R, \delta^{p}\right)$, Theorem 8 says that r. gl. $\operatorname{dim} . R\left[\theta^{p}\right]=n+1$. Observing that $R[\theta]$ is a free right and left $R\left[\theta^{p}\right]$ module, we obtain r. gl. dim. $R[\theta] \geq n+1$ from [1, Lemma 2.b.4], and by Proposition 3 we are done.

Combining Theorems 5 and 8 immediately yields the following formula:

Theorem 10. Let $R$ be a noetherian Ritt algebra with g1. $\operatorname{dim} R=n<\infty$. If $k=\sup \left\{\operatorname{pd}_{R}(R / P) \mid P\right.$ is a prime differential ideal of $\left.R\right\}$, then r. gl. $\operatorname{dim} . R[\theta]=\max \{n, k+1\}$.

4. Applications and remarks. (a) The Weyl algebra $A_{1}(S)$ over a ring $S$ is just $S[x][\theta]$, where we use the derivation $\delta=\partial / \partial x$ on $S[x]$. If $S$ is a field of characteristic 0 , then r. gl. $\operatorname{dim} . A_{1}(S)=1$, while if $S$ is a field of positive characteristic, then r. gl. $\operatorname{dim} . A_{1}(S)=2[9$, Theorem, p. 345]. We generalize these results as follows:

Theorem 11. Let $S$ be a commutative noetherian ring with gl. $\operatorname{dim} . S=$ $n<\infty$. If $S$ is an algebra over the rationals, then r. gl. $\operatorname{dim} . A_{1}(S)=n+1$, while if $S$ has positive characteristic, then r. gl. $\operatorname{dim} . A_{1}(S)=n+2$.

Proof. Let $R$ denote the ring $S[x]$ with the derivation $\partial / \partial x$.

Assuming that $S$ is an algebra over the rationals, let $P$ be any differential ideal of $R$, and set $J=P \cap S$. It is easy to see that $P=J R[x]$, whence $\operatorname{pd}_{R}(R / P) \leq \operatorname{pd}_{S}(S / J) \leq n$. According to Theorem 5, r. gl. dim. $A_{1}(S)=n+1$.

Now assume that $S$ has positive characteristic. In view of [6, Part III, Theorems 11, 13], $S$ is a regular noetherian ring, hence [7, Theorem 168] says that $S$ is a finite direct product of domains. Thus we need only consider the case when $S$ is a domain, and here the characteristic of $S$ must be a prime $p$.

In view of [7, Exercise 7, p. 53], we must have $\operatorname{pd}_{S}(S / P)=n$ for some prime ideal $P$ of $S$. Let $Q=P+x R$, which is a prime ideal of $R$ such that $R / Q \cong S / P$. Now $\operatorname{pd}_{R}(R / Q)=n+1$ by [6,P art III, Theorem 3], and it is clear that $\delta^{p}(Q) \subseteq Q$, hence Corollary 9 says that r. gl. dim. $A_{1}(S)=n+2$.

(b) For any ring $S$, one can define a ring $F_{1}(S)=S[[x]] \cdot[\theta]$ using the derivation $\partial / \partial x$ on $S[[x]]$. Proceeding as in Theorem 11, we obtain the following results, of which the first part is a special case of [1, Theorem 4.2].

Theorem 12. Let $S$ be a commutative noetherian ring with gl. $\operatorname{dim} . S=$ 
$n<\infty$. If $S$ is an algebra over the rationals, then r. gl. $\operatorname{dim} . F_{1}(S)=n+1$, while if $S$ has positive characteristic, then r. gl. $\operatorname{dim} . F_{1}(S)=n+2$.

(c) [4, Theorem 2.6] says that if $R$ is a commutative noetherian integral domain (not a field) with finite Krull dimension, and if $\delta$ is a derivation on $R$ such that $R[\theta]$ is a simple ring, then r. gl. $\operatorname{dim} . R[\theta]=$ gl. $\operatorname{dim} . R$. As noted in [4, Lemma 2.4], it follows from these hypotheses that $R$ is a Ritt algebra and that the only differential ideals in $R$ are 0 and $R$. Thus for the case gl. $\operatorname{dim} . R<\infty$, this theorem is a consequence of Theorem 5 . For the case gl. $\operatorname{dim} . R=\infty$, however, the proof of this theorem relies on the inequality gl. $\operatorname{dim} . R \leq$ r. gl. $\operatorname{dim} . R[\theta]$ and so is open to question.

(d) [3, Theorem 2] is another theorem which relies on the inequality gl. $\operatorname{dim} . R \leq \mathrm{r} . \mathrm{gl}$. dim. $R[\theta]$, in the infinite-dimensional case and so is open to question. In the finite-dimensional case, this result is an easy consequence of Theorem 8 .

(e) We conclude by noting that Theorems 5 and 10 may fail in cases of positive characteristic. For example, let $F$ be any algebraically closed field of positive characteristic, and set $R=F[x], \delta=\partial / \partial x$. Since the maximal ideals of $R$ are generated by linear polynomials, none of them can be differential ideals. Thus we have $\operatorname{pd}_{R}(R / P)=0<\mathrm{gl}$. dim. $R$ for all prime differential ideals $P$ of $R$, whereas Theorem 11 says that

$$
\text { r. gl. } \operatorname{dim} . R[\theta]=2>\text { gl. } \operatorname{dim} . R \text {. }
$$

Perhaps Theorems 5 and 10 may be made to hold in general by using more differential ideals $P$ than just the prime ones.

Added in proof.

(f) Some results related to those in this paper have appeared in S. M. Bhatwadekar, On the global dimension of Ore-extensions, Nagoya Math. J. 50 (1973), 217-225. Bhatwadekar's Theorem 2.3 gives the rational case of our Theorem 11, and his Theorem 1.1 can be obtained as a direct consequence of our Theorem 5 .

(g) Other results related to the present paper appear in G. S. Rinehart and A. Rosenberg, The global dimension of Ore extensions and Weyl algebras, Communications in Algebra (to appear). Their Theorem 2.6 generalizes our Theorem 11 to the case of Weyl algebras of arbitrary degree.

(h) In a sequel to the present paper, we generalize the results given here to the case of a commutative noetherian ring with a finite collection of commuting derivations. Also, we answer the question raised in Remark (e): Theorem 10 holds for commutative noetherian differential rings $R$ with 
gl. $\operatorname{dim} . R<\infty$, provided $k$ is set equal to the supremum of $\mathrm{pd}_{R}(R / P)$ where $P$ ranges over all primary differential ideals of $R$.

\section{REF ERENCES}

1. J.-E. Björk, The global homological dimension of some algebras of differential operators, Invent. Math. 17 (1972), 67-78.

2. H. Cartan and S. Eilenberg, Homological algebra, Princeton Univ. Press, Princeton, N. J., 1956. MR 17, 1040.

3. N. S. Gopalakrishnan and R. Sridharan, Homological dimension of Ore-extensions, Pacific J. Math. 19 (1966), 67-75. MR 34 \#223.

4. R. Hart, Krull dimension and global dimension of simple Ore-extensions, Math. Z. 121 (1971), 341-345. MR 45 \#6811.

5. I. Kaplansky, An introduction to differential algebra, Actualités Sci. Indust., no. 1251, Hermann, Paris, 1957. MR 20 \#177.

6. - Fields and rings, Univ. of Chicago Press, Chicago, Ill., 1969. MR $42 \# 4345$.

7. I. Kaplansky, Commutative rings, Allyn and Bacon, Boston, Mass., 1970. MR $\mathbf{4 0}$ \#7234.

8. R. Rentschler and P. Gabriel, Sur la dimension des anneaux et ensembles ordonnés, C. R. Acad. Sci. Paris Sér. A-B 265 (1967), A712-A715. MR 37 \#243.

9. G. S. Rinehart, Note on the global dimension of a certain ring, Proc. Amer. Math. Soc. 13 (1962), 341-346. MR 25 \#1196.

10. J-E. Roos, Detérmination de la dimension homologique globale des algèbres de Weyl, C. R. Acad. Sci. Paris Sér. A-B 274 (1972), A23-A26. MR 45 \#1995.

11. The Weyl algebras are Gorenstein rings. Generalizations and applications (to appear).

12. L. Silver, Noncommutative localizations and applications, J. Algebra 7 (1967), 44-76. MR $36 \# 205$.

DEPARTMENT OF MATHEMATICS, UNIVERSITY OF UTAH, SALT LAKE CITY, UTAH 84112 